\title{
The Algorithm of Measuring in Close-Range Photogrammetry based on Grid
}

\author{
Wanli Xu, Zhun Liu, Huiyu Nie \\ Department of Information Engineering \\ Academy of Armored Force Engineering \\ Beijing,100072,China \\ E-mail: xuwanli631101@sina.com
}

\begin{abstract}
The Algorithm of Measuring in Close-Range Photogrammetry based on Grid

[Purpose] Due to the existence of various error sources in the close-range photogrammetry, the traditional photogrammetry algorithm cannot completely eliminate system error. The measurement precision often cannot reach the requirement and the calculation process is complicated. In order to solve this problem, a new measuring algorithm called grid measuring algorithm is presented in this papers. [Method]We structures an ideal grid measuring space according to image point of the fixed point. Then the interested object points are mapped to the grid measuring space by the image points. We can get the position by fusing the value according to the distance to the nodes.For illustration,a photographic example is utilized to show the feasibility of the grid measuring algorithm.[Results] The experimental results show that the error of grid measuring algorithm is controlled within half pixels. [Conclusions]The positioning precision of this algorithm is obviously superior to the traditional algorithm and the calculation is simple.
\end{abstract}

Keywords- close-range photogrammetry; grid measuring space; grid measuring algorithm

\section{INTRODUCTION}

Photogrammetry is to calculate the position of the object point according to its image point by photographing. Closerange photogrammetry is the photogrammetry that the distance is close. If the algorithm of close-range photogrammetry is appropriate, the measurement error could be controlled within $0.1 \mathrm{~mm}[1]$. So close-range photogrammetry has high positioning accuracy and it can be used for aerospace workpiece machining control as well as to other measuring instrument inspection correction. It has high practical value. But there are various error sources such as the distinction of focal distances of the two cameras, the bias of cameras, the errors of fixed points and so on in the closerange photogrammetry. The positioning precision is affected directly. The traditional photogrammetry algorithms usually calculate the parameters and correct them one by one. But it cannot completely eliminate system error and the calculation process is complicated. So the research of photogrammetry algorithm is the key point to improve the positioning precision.

Because the distance of close-range photogrammetry is close, the controlling points and object points could be fixed by manual work. It is beneficial to eliminate system error[2].

\section{PRINCIPLE OF PHOTOGRAMMETRY}

Assuming that the coordinate system is S1-XYZ,it is shown in Figure 1. Its origin point is S1 which is the focal point of left camera and its $\mathrm{Y}$ axis is $\mathrm{S} 1 \mathrm{O} 1$ which is the main optical axis of left camera and its $\mathrm{Z}$ axis is the plumb line passing through S1.The connecting line S1S2that links the focal points of two cameras is the baseline $\mathrm{B}$. The focal distance of camera is $\mathrm{f}$. Photo is behind focal point in practice. We can evolve photo before focal point as shown in Figure 1.

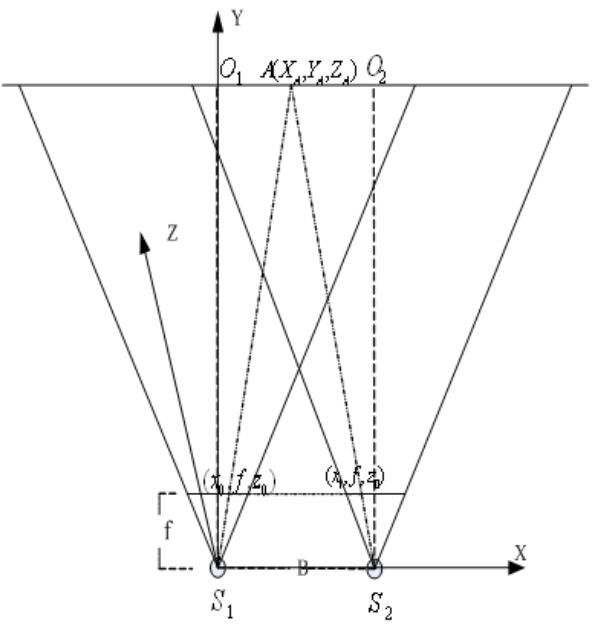

Figure 1. Coordinate system

Assuming that the coordinate of object point $A$ is $\left(X_{A}, Y_{A}, Z_{A}\right)$, the coordinates of corresponding image points in left and right photos are $\left(x_{0}, f, z_{0}\right)$ and $\left(x_{1}, f, z_{0}\right)$. Because the object point, the image point and the focal point of camera are on the same line, we can get two equations of straight lines that are followed as below[3][4]:

$$
\left\{\begin{array}{l}
\frac{x-x_{0}}{X_{A}-x_{0}}=\frac{y-f}{Y_{A}-f}=\frac{z-z_{0}}{Z_{A}-z_{0}} \\
\frac{x-x_{1}}{X_{A}-x_{1}}=\frac{y-f}{Y_{A}-f}=\frac{z-z_{0}}{Z_{A}-z_{0}}
\end{array}\right.
$$


As the focal points of two cameras are on the lines above-mentioned and their coordinates are $(0,0,0),(B, 0,0)$.we can get the results followed as below[5]:

$$
\left\{\begin{array}{l}
X_{A}=\frac{B}{B-p} x_{0}=\frac{Y_{A}}{f} x_{0} \\
Y_{A}=\frac{B}{B-p} f \\
Z_{A}=\frac{B}{B-p} z_{0}=\frac{Y_{A}}{f} z_{0} \\
p=x_{1}-x_{0}
\end{array}\right.
$$

A line segment that its length is $\mathrm{L}$ on the object plane which is parallel to the photo plane and its distance far away from the focal point is $\mathrm{Y}$ has the relation with the corresponding pixel segment that its length is 1 in the photo as below:

$$
Y / f=L / l
$$

We can get the length of base line by equations (1) and (2) as below:

$$
B=p /(1-l / L)
$$

In order to calculate the focal distance of camera $f$, we take steps as below. Assuming that there is a board that its distance far away from the focal point is $\mathrm{Yi}$, we call it as $\mathrm{i}$-th plane. Then move the board to the plane called j-th plane that its distance far away from the focal point is $\mathrm{Yj}$ along the main optical axis. Find two sign points that its distance is $\mathrm{L}$ in the i-th plane. Calculate the pixels li that the two sign points space. Then find the two corresponding sign points in the j-th plane and calculate the pixels lj. So the lengths of the two sign points in the i-th plane and in the j-th plane are $\mathrm{li}^{*} \mathrm{dx}, \mathrm{lj}{ }^{*} \mathrm{dx}$, where $\mathrm{dx}$ is the length of every pixel.

According to equation (2), we know that,

$$
\begin{aligned}
& Y_{i} / f=L /\left(l_{i} \times d x\right) \\
& Y_{j} / f=L /\left(l_{j} \times d x\right)
\end{aligned}
$$

And (5)-(4), we can obtain the focal distance of the camera.

$$
f=\frac{Y_{j}-Y_{i}}{L \times\left(\frac{1}{l_{j} \times d x}-\frac{1}{l_{i} \times d x}\right)}
$$

\section{GRID MEASURING ALGORITHM}

We structure a grid measuring space. That is to say, we structure a ideal grid measuring space according to the image points of the sign points. And then map the object point to the grid measuring space through its corresponding image point. At last, we can get the position by fusing the value according to the distance to the nodes of the grid net. We illustrate it through an example.

Firstly, we structure a grid measuring plane. As is shown in Figure 2, there is an auxiliary glass board that its area is $420 \mathrm{~mm} \times 420 \mathrm{~mm}$. There is a sign point every 20 $\mathrm{mm}$ interval. The sign points structure a grid net. The plane where the glass board locates is the 0 -th plane. Then take out the sign points of the row that close to the main optical axis and calculate the interval pixels 10 that every two sign points space according to the first sign point and the last sign point. Make the first sign point that close to the main optical axis as the start point and 10 as the interval to structure the grid measuring plane.

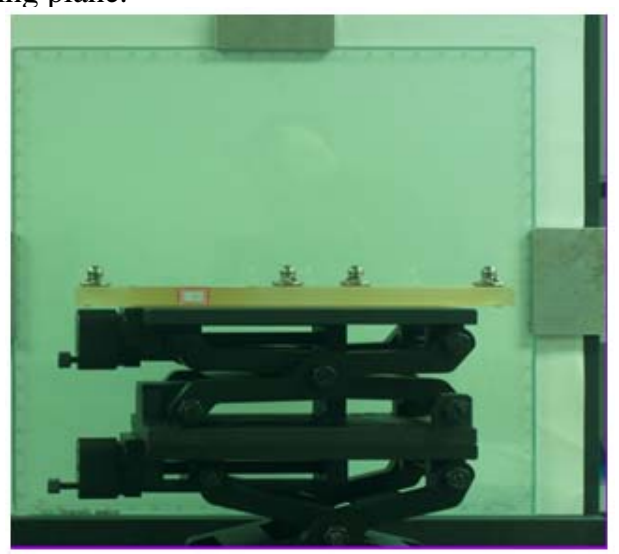

Figure 2. Experiment environment

Then, we structure the grid measuring space. According to equation (4), the horizontal distance of an arbitrary object point to the main point $\mathrm{L}$ has the relation with its pixel coordinate $x_{i}: Y_{i} \times x_{i} \times d x=L \times f, i=0,1,2, \ldots, N$. So we can get the equation as below:

$$
Y_{i} \times x_{i}=Y_{j} \times x_{j}, i, j=0,1,2, \ldots, N
$$

According to equation (7), if we know the distances of an arbitrary plane and 0 -th plane far away from the focal point and the horizontal pixel coordinate, we can get the horizontal pixel coordinate in the plane above-mentioned. So we can obtain the other plane through 0-th plane. At last we get the grid measuring space.

After structuring the grid measuring space, map the image points to the grid measuring space. Map the sign point $O_{i j}$ that locates at the $i$-th row and $j$-th column in the photo, $i, j=1,2 \ldots, 21$, to the sign point $O_{i j}$ that locates at the $i$-th row and $j$-th column in the grid measuring space. Map other points in the photo to the grid measuring space by fusing the value according to the distance to the nodes of the grid net as below:

$$
\begin{aligned}
& X=X_{i j}+\left(x-x_{i j}\right) \times l /\left(x_{i j+1}-x_{i j}\right) \\
& Z=Z_{i j}+\left(Z-Z_{i j}\right) \times l /\left(z_{i+1 j}-z_{i j}\right)
\end{aligned}
$$

Where $(x, z)$ is the coordinate of the image point $o$ between the image points $O_{i j}, O_{i j+1}, O_{i+1 j}, O_{i+1 j+1} \quad, \quad\left(x_{i j}, Z_{i j}\right),\left(x_{i j+1}, Z_{i j+1}\right)$, $\left(x_{i+1 j}, Z_{i+1 j}\right),\left(x_{i+1 j+1}, Z_{i+1 j+1}\right)$ are the coordinates of the four image points in the photo. $\left(X_{i j}, Z_{i j}\right)$ is the coordinate of the point which locates at the $i$-th row and $j$-th column in the grid measuring space and $l$ is the pixel interval. Then $(X, Z)$ is the coordinate that the object point $o$ maps to the grid 
measuring space. At last, we can calculate the position of the object point $o$ according to equation (1).

\section{EXPERIMENT}

We measure a standard instrument that is in common use international in the lab of as shown in Figure 2. The standard instrument demarcates three distances between the circles of balls. (see Table I )Locate the standard instrument at ten different positions in the same distance far away from the camera and then photograph it. We photographed the glass board and the standard instrument at 0 -th plane and 1-th plane with the left and right cameras. The pixel area of photo is $8176 \times 6132$. The length of every pixel is $0.00599315 \mathrm{~mm}$.

According to equation (6), we can calculate the focal distance of camera is $79.3019 \mathrm{~mm}$.

Map the circles of balls to the grid measuring space and then calculate the distances between the circles of balls shown in Table I.

\section{TABLE I. TABLEI. EXPERIMENT RESULTS}

\begin{tabular}{|c|c|c|c|}
\hline $\begin{array}{c}\text { Marked } \\
\text { Distance }\end{array}$ & $\mathbf{1 5 0 . 0 0 3 4}$ & $\mathbf{3 0 0 . 0 0 3 8}$ & $\mathbf{9 9 . 9 9 0 9}$ \\
\hline Position 1 & 150.022905 & 299.985872 & 100.081973 \\
\hline Position 2 & 150.024882 & 299.98099 & 100.013289 \\
\hline Position 3 & 150.131724 & 300.086703 & 100.119358 \\
\hline Position 4 & 150.101204 & 300.011683 & 100.068886 \\
\hline Position 5 & 150.038428 & 299.991265 & 100.162784 \\
\hline Position 6 & 150.088656 & 299.942022 & 100.075656 \\
\hline Position 7 & 149.955386 & 300.026163 & 100.139414 \\
\hline Position 8 & 150.077643 & 299.941602 & 99.904372 \\
\hline Position 9 & 149.921781 & 299.920793 & 100.051582 \\
\hline Position 10 & 150.080424 & 299.957109 & 100.036558 \\
\hline Average & 150.044303 & 299.984420 & 100.065387 \\
\hline Error & 0.0409 & 0.0194 & 0.0745 \\
\hline $\begin{array}{c}\text { Standard } \\
\text { Deviation }\end{array}$ & 0.065871 & 0.048801 & 0.072979 \\
\hline
\end{tabular}

The average is the average of distances of the circles of the balls at ten different positions and the standard deviation is the standard deviation of distances of the circles of the balls at ten different positions. The error is the deviation between the average and the marked distance. According to the distance of the standard instrument far away from the camera and the focal distance of camera, we can conclude that the error of grid measuring algorithm is controlled within half pixels.

\section{SUMMARY}

This paper presents a new measuring algorithm called grid measuring algorithm. It structures an ideal grid measuring space according to image point of the fixed point. Then the interested object points are mapped to the grid measuring space by the image points. We can get the position by fusing the value according to the distance to the nodes. The positioning precision of this algorithm is obviously superior to the traditional algorithm and the calculation is simple. The experimental results show that the error of grid measuring algorithm is controlled within half pixels.

\section{REFERENCES}

[1] Junjian Lin, Guihua Chang. Photogrammetry. Beijing:National Defence Industry Press,2006.

[2] Zuxun Zhang, Jianqing Zhang. Digital Photometry. Wuhan:Wuhan University Press,1997.

[3] Ying Chen. Remote Sensing Image Digital Photogrammetry. Tongji University Press,2003.

[4] Zhoucheng Li. Intelligent Image Processing Technology. Beijing:Electronic Industry Press,2004.

[5] Liangzheng Xia. Digital Image Processing. Southeast University Press,1999. 